\title{
Un Caso Real de Combate al Fracaso en la Educación Pública: Una Cuestión de Acompañamiento, Liderazgo y Cultura Organizacional
}

\author{
A Real Case of Dropout Prevention in Public Education: A \\ Matter of Follow-up, Leadership and Organizational Culture
}

Adriana Aristimuño ${ }^{1 *}$ Juan Pablo Parodi 2

${ }^{1}$ Universidad Católica del Uruguay. ${ }^{2}$ Colegio San José (Libertad)

\begin{abstract}
Se estudia la forma de trabajo pedagógico de un centro secundario de gestión estatal que en los últimos 10 años logra resultados sobresalientes, en términos de logros de aprendizaje y retención de estudiantes. Las preguntas que guiaron el trabajo apuntaron a identificar dichas estrategias y a describir el rol que tienen tanto la dirección del centro como la cultura organizacional en su definición. Se realizó un estudio de caso, que implicó más de 70 horas en el terreno, 30 entrevistas, análisis de documentos (libretas de profesores, actas de reuniones), y observación (ambiente físico, interacciones, reuniones, recreos, clases de tutorías). El centro elegido desarrolla un conjunto amplio de estrategias de acompañamiento de los aprendizajes, el liderazgo de la dirección coloca los aprendizajes en un lugar central y se focaliza en la solución de problemas endémicos de gestión, y existe una cultura organizacional integrada, con marcada presencia de las familias de los alumnos. El trabajo dialoga con la literatura sobre abandono escolar temprano, uno de los problemas más importantes que enfrentan las sociedades contemporáneas especialmente en el nivel de la educación secundaria, y hace aportes originales en la identificación de estrategias de acompañamiento de trayectorias de estudiantes en riesgo de fracaso.
\end{abstract}

Descriptores: Fracaso escolar, Deserción escolar, Repetición, Orientación pedagógica, América Latina.

The study makes an in-depth investigation in the pedagogic model of a public secondary school that in the last 10 years has attained outstanding good results in terms of students' learning and dropout prevention. The main research questions aimed to identify the follow-up strategies, to describe the leadership style, and the organizational culture, including the role of the families in it. The method used was a case study that involved more than 70 hours of field work, 30 interviews, document analysis (teacher's registrations, meetings follow-ups) and observation (of the building, main interactions, meetings, recess time, support classes and tutoring activities). The chosen school showed a varied range of follow-up activities of the learning process of the students, as well as an instructional leadership that stresses the centrality of learning issues, a prompt attitude of the principal to solve administrative issues such as teacher absenteeism and an integrated school culture in which the students' families are visible and active. This study dialogues with research on early school leaving, one of the main issues contemporary societies face, especially at the secondary school's level, and makes an original contribution to the identification of school level follow-up strategies of the learning processes of at-risk students.

Keywords: Academic failure, Dropping out, Grade repetition, Educational guidance, Latin America.

*Contacto: aaristim@ucu.edu.uy

ISSN: $1696-4713$

www.rinace.net/reice/

revistas.uam.es/reice
Recibido: $\quad 3$ de agosto 2017

$1^{\text {a }}$ Evaluación: 5 de septiembre 2017

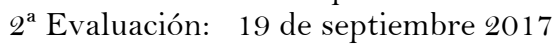

Aceptado: $\quad 22$ de septiembre 2017 


\section{Introducción}

La superación del abandono escolar prematuro (AEP), es una prioridad de la política educativa actual (Casquero y Navarro, 2010; Comisión Europea, 2013; Escudero y Martínez-Domínguez, 2012; Pedró, 2012; Rumberger, 2011). En algunos continentes, como África o América Latina, y dada la asociación que el fenómeno tiene con la inequidad, el problema se vuelve particularmente crítico y desafiante (Guimaraes de Castro, 2011; Pont, 2014). Europa se ha planteado como objetivo que a los 24 años de edad todos sus ciudadanos deberán haber completado la educación secundaria, y en el marco de la estrategia Europa 2020, se ha propuesto como meta que para esa fecha solo quede fuera de dicho logro el 10\% de su población (Comisión Europea, 2010; Pedró, 2012).

Diferentes autores han intentado identificar los numerosos factores que inciden en que el abandono todavía sea un fenómeno esquivo de superar. España se encuentra en niveles elevados, superiores al 20\%, muy lejos de la meta europea. América Latina comparte esa realidad.

En los últimos años se ha desplazado la atribución causal sobre el fracaso escolar: lentamente se va tomando conciencia de que no se trata tan solo del fracaso de trayectorias escolares individuales, sino que está determinado por un conjunto de factores que inciden en forma negativa en los aprendizajes, factores provenientes tanto de la esfera socio-familiar del estudiante, como de las características del servicio educativo (Aristimuño, 2015). En esta transformación han influido la conceptualización de la educación como un derecho, y la noción de educación inclusiva, que a partir de posturas como la de Ainscow (2005), proponen que son las condiciones y características del servicio educativo las que facilitan o impiden que se cumpla con este derecho. Este artículo presenta hallazgos sobre la forma en que un centro educativo, sin contar con recursos ni programas específicos para ello, logra resultados excepcionales en términos de retención y aprendizajes, y pone foco en tres conjuntos de factores: el tipo de liderazgo que lo conduce, la cultura organizacional y las estrategias de acompañamiento de los aprendizajes de los alumnos.

\section{Fundamentación teórica}

Los procesos por los cuales los estudiantes pierden interés, faltan frecuentemente a clase y abandonan la escuela, muestran que se trata de procesos en los cuales intervienen múltiples factores, pero entre los cuales la soledad, el anonimato en la institución, y la pérdida de sentido del acto educativo son centrales en la percepción de los estudiantes (Mena, Fernández Enguita y Riviere, 2010; Rué, Amela y Buscarons, 2003). Investigaciones de la región latinoamericana confirman que, en la adolescencia, y en forma creciente conforme aumenta la edad, las principales causas para abandonar los estudios no son las socioeconómicas, sino las relativas a las características de la oferta educativa (SITEAL, 2009). Ya no es un fenómeno de los sectores más desfavorecidos en lo socioeconómico, sino que en forma creciente los estudiantes de las clases medias también pierden interés en lo que la escuela les ofrece.

Para abordar el tema se requiere un enfoque amplio, que dé cuenta de los diferentes aspectos que inciden en los logros de los aprendizajes. Recientes estudios referidos al tema de logros y fracasos educativos, proponen diferentes factores, tales como los del 
contexto (de las políticas), los escolares (que incluyen desde el proyecto de centro hasta las relaciones entre los actores), los relativos al aula (donde resultan cruciales los materiales educativos y las estrategias de enseñanza), y los del alumnado (Santos-Rego, Godás-Otero y Lorenzo-Moledo, 2012).

La literatura sobre las escuelas eficaces y la de la mejora escolar se han ocupado del tema de los resultados de aprendizaje, y los factores que inciden en su mejora. Desde los primeros trabajos sobre escuelas eficaces (Brookover et al., 1979; Edmonds, 1979; Rutter et al., 1979), ya se identificaba a estos factores, tales como la conducción de los centros, la gestión concentrada en la enseñanza, su cultura escolar, altas expectativas, disciplina ordenada y foco en el lenguaje y la matemática. En la literatura sobre mejora, se agregan otros aspectos, como el contexto o la constitución de equipos (Bryk et al., 2009; Murillo y Krichesky, 2015). Además, puede afirmarse que ambas corrientes se integran en la de la mejora de la eficacia escolar (Murillo, 2005, 2007). Hoy día, recientes desarrollos ponen más énfasis en la mejora de los aprendizajes, lo que ha llevado la atención a lo que ocurre en las aulas, a la necesidad de contar con liderazgos distribuidos, y a la constitución de comunidades de aprendizaje por parte de los profesores en centros educativos que se hacen responsables por los resultados (Bolívar, 2015; Murillo et al., 2015).

La literatura sobre eficacia escolar ha profundizado en forma reciente sobre los factores que inciden tanto en el logro de resultados mejores de lo esperado según el contexto (escuelas "eficaces positivas") como en el logro de resultados peores, en el sentido que los estudiantes no aprenden lo esperable de acuerdo a dicho contexto ("eficaces negativas"). En este último sentido, se cuenta con trabajos que han identificado los factores de ineficacia escolar (Murillo, Hernández-Castilla y Martínez-Garrido, 2014), entre los que se destacan un clima negativo, sensación de desánimo y bajas expectativas, falta de compromiso y motivación, así como escaso trabajo en equipo de los profesores, una dirección ausente o autoritaria, poca implicación de las familias, procesos de enseñanza reproductivos e instalaciones inadecuadas. Algunas revisiones de literatura (Aristimuño, 2015) sistematizan recientes hallazgos que permiten ubicar las diversas causas que inciden en el fracaso escolar, desde un nivel más general, como el macro curricular, hasta otros de nivel más focalizado y concreto, como el centro educativo y el aula. En el nivel curricular, pueden evitar altos niveles de fracaso una adecuada articulación entre la primaria y la secundaria, una bifurcación curricular lo más tardía posible, así como la eliminación de la repetición (Pont, 2014). En los niveles de centro y aula, escuelas pequeñas y grupos no demasiado numerosos (Faubert, 2012), liderazgos que hagan foco en los aprendizajes y contribuyan a la construcción de comunidades profesionales de aprendizaje entre los profesores, así como establecer fuertes conexiones culturales con los estudiantes, poner a éstos a trabajar en grupos, utilizar sistemáticamente la evaluación para retroalimentar la enseñanza, y estrategias relativas a la secuencia adecuada de los contenidos, destacan en la literatura de abatimiento del fracaso (Faubert, 2012).

En forma reciente la OCDE (2016) ha propuesto, a partir de su programa internacional de evaluación de aprendizajes, cuáles serían los factores asociados a los rendimientos más bajos. En el cuadro 1 se presenta una síntesis simplificada y adaptada de los mismos, distinguiendo entre la órbita de los alumnos, la de las escuelas y la del sistema educativo. 
En paralelo al reciente crecimiento económico de la década pasada, al abatimiento de la pobreza y a mejoras sustantivas en la distribución de la riqueza, América Latina ha experimentado importantes mejoras también en sus logros educativos. En un estudio comparado de siete países de la región, se constata que "entre los años 2000 y 2012, América Latina fue la región que más aumentó sus resultados en las pruebas PISA y la que más aumentó su tasa de escolarización de la educación secundaria” (Rivas, 2015, p. 18).

Cuadro 1. Factores asociados al rendimiento bajo

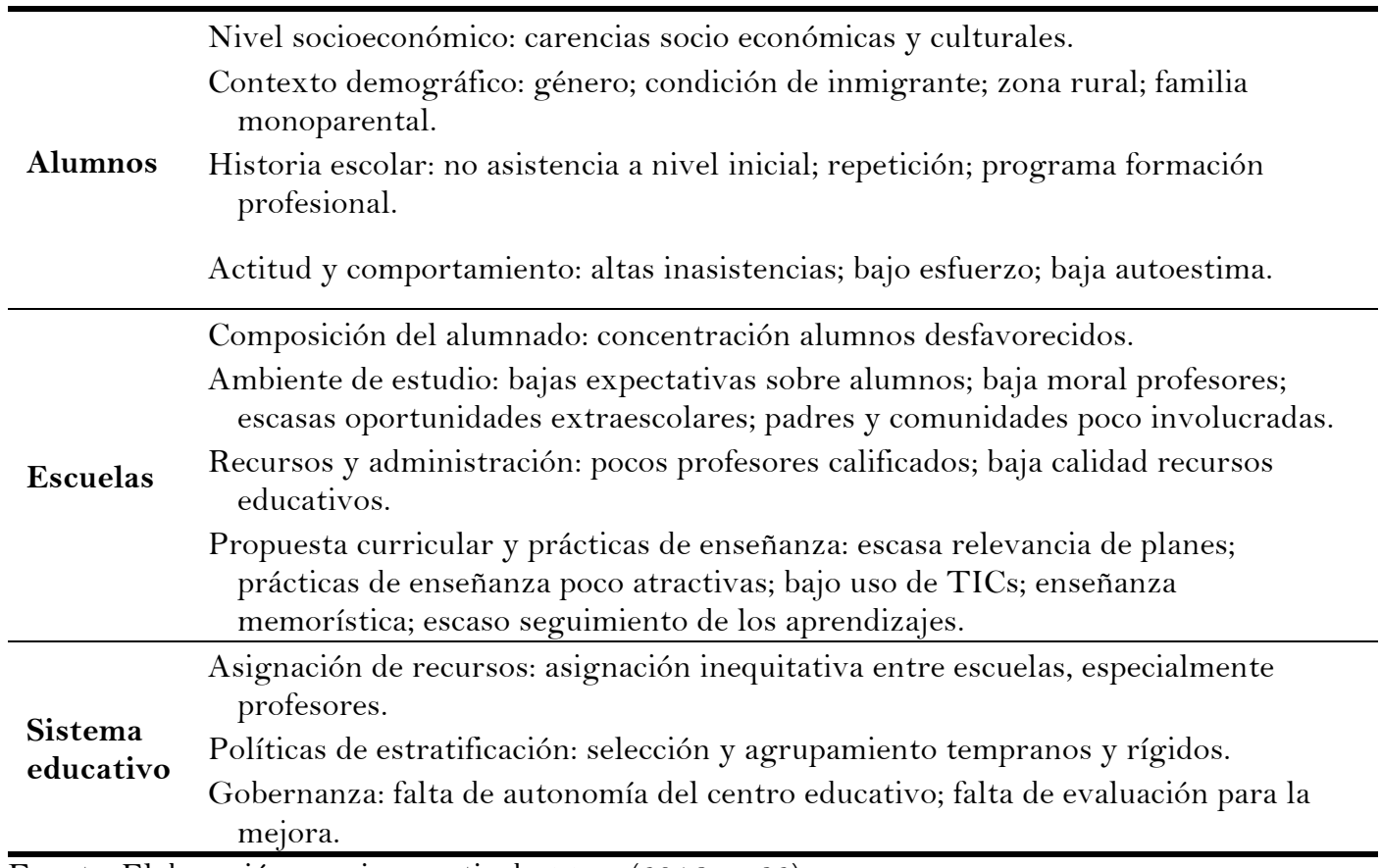

Fuente: Elaboración propia a partir de OCDE (2016, p. 32).

Sin embargo, en el marco de este contexto favorable, un pequeño país con una sociedad mayoritariamente integrada en torno a una sola lengua, con buena infraestructura de comunicaciones y la expansión universal de su educación primaria, ha experimentado un visible deterioro de sus resultados educativos, particularmente en el tramo secundario. En el referido trabajo de Rivas (2015, p. 214) se muestra que Uruguay en 2011 tenía, en su población de 17 años, la menor proporción de jóvenes cursando en la edad teórica $(14,4 \%)$ de los siete países, así como el segundo peor resultado de población cursando con sobre edad $(47,3 \%)$, y también el segundo peor resultado en población que no asiste a la educación formal (38,2\%). Son resultados significativamente malos, dada su situación económica favorable, sus características ya señaladas y su tradición de alta calidad educativa.

Recientemente, algunos estudios realizados en Uruguay se han dedicado a analizar algunos aspectos de esta realidad, destinados a identificar las causas del abandono (Aristimuño, 2016; de Melo, Failache y Machado, 2015), o a describir las trayectorias de los alumnos que no culminan la educación secundaria (Cardozo, 2016), que son casi el $60 \%$. Numerosos programas se han puesto en marcha en diferentes países para combatir el fracaso, de lo cual Uruguay no es excepción, donde las iniciativas funcionan en los niveles de educación primaria y secundaria (general y técnica). Se trata e iniciativas tanto de prevención del fracaso, orientados al seguimiento y detección temprana de 
alumnos en riesgo, como de rescate de desertores: maestros comunitarios, aulas comunitarias, liceos con tutorías, tránsito educativo, compromiso educativo, formación profesional básica, entre los más significativos. Varios de ellos han sido evaluados por las autoridades del sistema educativo, así como por estudios académicos independientes. Existe consenso en que han dado resultados magros y que su alcance numérico es muy limitado (Instituto Nacional de Evaluación Educativa, 2014).

Todo lo anterior sostiene la pertinencia del presente estudio, no solo porque el tema del fracaso se ha convertido en una prioridad para las autoridades y un problema de difícil superación, sino porque su foco se ubica en las dinámicas de seguimiento de los aprendizajes, en la organización escolar y en el rol de las familias, es decir, en dinámicas internas al centro educativo. El estudio fue realizado en un centro educativo promedio tanto en su composición social y su dotación material en equipamiento, como en las características de sus recursos humanos. Por ello, resulta muy pertinente conocer sus dinámicas de trabajo, ya que allí puede radicar la clave de sus buenos resultados educativos. En términos del cuadro 1, la investigación hace foco en los factores del nivel de escuela identificados como ambiente de estudio y propuesta curricular y prácticas de enseñanza.

El estudio recoge una importante acumulación conceptual en el área del acompañamiento escolar, aspecto vinculado al área más general de la orientación escolar. Estudios recientes han mostrado la importancia de las actividades de orientación como instrumento de superación del fracaso (González Berruga, 2016). Una idea central del presente estudio es que el énfasis para superar el fracaso, no está tanto en las características de la escuela o del alumnado, sino en la calidad de los espacios educativos como espacios de oportunidad. Utilizando este concepto, Rué, Amela y Buscarons (2003) señalan que las principales condiciones que provocan el absentismo escolar -como sinónimo de autoexclusión-, son el sentimiento de pérdida de autoestima del estudiante, y el desencuentro entre sus intereses y los de la escuela. En este estudio se utiliza una noción de acompañamiento que implica: conocimiento del estudiante y su entorno, integración de su familia a la vida del centro escolar, trabajo coordinado entre los educadores, una atención a la educación integral no solo en su componente intelectual, definición de estrategias concretas de apoyo, adaptaciones curriculares de los docentes y un registro sistemático de las intervenciones (Parodi, 2015). Un estudio de evaluación de impacto realizado en Uruguay señala la importancia del acompañamiento de los procesos formativos de los adolescentes, en el logro de excepcionales resultados, y también en la ampliación de las expectativas sobre la continuación de los estudios, tanto de los estudiantes como de sus familias (Balsa y Cid, 2014).

Otro elemento conceptual central en el trabajo es el del liderazgo de la dirección escolar y su relación con este acompañamiento. Es algo ya aceptado que el liderazgo pedagógico de un director, que coloca los temas de aprendizaje en el centro de su gestión, constituye una guía fundamental de los procesos formativos. Se ha señalado repetidamente (Bolívar, 2015; Harris, 2009) la importancia de distribuir dicho liderazgo a los equipos y a los docentes, aunque se reconoce que se trata de un tipo de liderazgo más exigente que el no distribuido. En estos liderazgos la clave es la confianza como base de la construcción de los procesos, lo que implica compartir miedos, atender sugerencias y asumir riesgos en conjunto (López-Yáñez y Lavié, 2010). También es importante considerar los liderazgos que desarrollan los maestros y su rol en los procesos de mejora, sobre todo en contextos de elevados niveles de fracaso (Harris y Muijs, 2005). Un reciente estudio realizado en 
Chile en 600 escuelas de educación básica (Horn y Murillo, 2016) profundizó en uno de los aspectos medulares de la dirección escolar, que es su incidencia en el compromiso de los docentes. Este compromiso es un factor mediador clave situado entre dicha dirección escolar y los aprendizajes de los alumnos: lo que el director hace sí afecta el compromiso de los docentes. Dos prácticas fueron identificadas como relevantes en el compromiso docente: que el director marque una dirección a seguir (en el sentido de la misión y la visión del centro) estructurando el centro educativo y creando las condiciones para que los docentes hagan su trabajo, y espacios concretos de apoyo pedagógico al trabajo de los docentes por parte de la jefatura técnico-pedagógica del centro.

Por último, el estudio también incorpora en su base conceptual, al rol de la familia del estudiante en la cultura organizacional. Una cultura organizacional de metas compartidas en torno a los aprendizajes de los alumnos es un sustrato positivo sobre el que construir estrategias de acompañamiento. Lo que los centros educativos hacen como organizaciones y la cultura que generan tiene efectos directos sobre las trayectorias de los estudiantes, hasta el punto de resultar en diferentes configuraciones en el tratamiento del riesgo de AEP, como han mostrado, entre otros, Tarabini y sus colaboradores (2015). En dicho estudio, las mismas políticas educativas generales aplicadas en cinco centros educativos resultan en cuatro lógicas de enfrentamiento del AEP diferentes: heterogeneidad, acompañamiento, omisión y expulsión.

El rol de las familias en la prevención del fracaso resulta crucial. Si bien algunos estudios se centran en la labor de las familias en el hogar, y lo que es necesario hacer para apoyar a los estudiantes (p. ej., Martínez Seijo, Rayón y Torrego, 2017), o en las competencias parentales concretas que son necesarias para prevenir el abandono (Martín Quintana et al., 2015), en este estudio el abordaje se centra en la integración de las familias a la vida del centro educativo como factor positivo en los aprendizajes de los estudiantes.

\section{Método}

El estudio se diseñó para responder tres preguntas:

- ¿Existen en este centro estrategias de acompañamiento de los aprendizajes de los alumnos que puedan ser identificadas, y si existen, cuáles son?

- ¿Qué tipo de cultura organizacional tiene el centro, y qué tipo de liderazgo ejerce la dirección?

- ¿Qué lugar ocupan las familias en el centro educativo, especialmente en la construcción de su cultura organizacional?

\section{Enfoque metodológico}

En función de estas preguntas, se optó por un enfoque metodológico de estudio de caso, en el entendido de que es el que mejor permite responderlas, con su abordaje comprehensivo de la realidad de una organización. El estudio de caso está especialmente recomendado por la literatura especializada para investigaciones en que la organización donde ocurre el fenómeno en estudio forma parte de los factores involucrados en el mismo: en este caso la organización escolar, y lo que ella se relaciona con la dirección escolar y la cultura del centro, resulta un componente fundamental (Yin, 2013). El estudio de caso, además, permite integrar diferentes técnicas de investigación, que la amplitud de las preguntas planteadas requiere. 
La selección del centro educativo se hizo en función de sus resultados de retención y logros de aprendizaje que, en forma sostenida en los 10 años previos a su selección, había estado entre los tres centros de educación secundaria con mejores resultados del país. Al respecto considérese que, al momento de su selección, la media nacional de estudiantes aprobados para centros secundarios se ubicaba por debajo de $70 \%$, mientras que el centro seleccionado siempre había estado por encima de $85 \%$, e incluso $90 \%$.

En el siguiente cuadro se expone información sobre el centro educativo seleccionado: la cantidad de estudiantes que sirve, el número de alumnos por grupo, y el principal indicador utilizado para su selección -el porcentaje de alumnos promovidos al cabo del año escolar, es decir, que aprueban el año con suficiencia y no repetirán curso-. Con el fin de proteger la identidad del centro, las cifras se han aproximado a decenas (para números enteros) y unidades (para porcentajes).

Cuadro 2. Información sobre matrícula y resultados educativos del centro seleccionado, años 2008-2014

\begin{tabular}{|c|c|c|c|c|c|c|c|}
\hline & 2008 & 2009 & 2010 & 2011 & 2012 & 2013 & $2015^{*}$ \\
\hline Alumnos matriculados en el centro & 370 & 380 & 360 & 330 & 330 & 330 & 290 \\
\hline Número de alumnos por grupo & 28 & 28 & 28 & 27 & 27 & 27 & 24 \\
\hline Porcentaje de alumnos promovidos & 86 & 86 & 89 & 91 & 91 & 87 & 95 \\
\hline $\begin{array}{l}\text { Porcentaje de alumnos promovidos, todo } \\
\text { el país }\end{array}$ & 70 & 68 & 68 & 67 & 68 & 69 & 73 \\
\hline
\end{tabular}

Nota: * El año 2015 no se utilizó para la definición del centro educativo por un tema de calendario: el trabajo de campo se realizó el año anterior, con información hasta 2013 inclusive. Se incluye la información relativa a 2015 solamente a efectos de completar la tendencia y aportar la última información disponible.

Fuente: Elaboración propia a partir de Monitor Educativo CES (http://www.anep.edu.uy/).

Como puede apreciarse, el principal indicador de resultados del centro -el porcentaje de alumnos promovidos- lo coloca en un lugar excepcional en relación al resto, logrando en 2015 un resultado de promoción que despega en más de $20 \%$ respecto de la media de alumnos promovidos en todo el país.

Por otra parte, el centro no tiene ninguna característica excepcional en sus variables estructurales tales como su planta física, su dotación de materiales educativos, las características de su plantel docente, o el contexto socioeconómico de la población estudiantil que sirve; más bien comparte algunas carencias típicas: falta un salón para aula, falta un laboratorio, falta un salón para reuniones, para lo que se usa la biblioteca. Por lo tanto, el centro comparte sus principales características con sus similares (centro que brinda el servicio educativo secundario en una ciudad de tamaño pequeño), es arquetípico del universo que integra, pero logra excepcionales resultados.

\section{Categorías de análisis}

El análisis siguió la lógica planteada por las preguntas de investigación, codificándose todas las entrevistas, los registros de observación y los contenidos de los documentos, con los mismos códigos y sub-códigos. Se diseñaron así cuatro familias de códigos vinculados con los cuatro conceptos centrales: estrategias de acompañamiento; liderazgo; cultura organizacional y familias. 
Estos cuatro conceptos guiaron el trabajo de análisis. A modo de ejemplo se incluyen a continuación las dimensiones y subcategorías de códigos (con su abreviatura en mayúscula y su explicación), utilizadas para el concepto acompañamiento escolar:

- Conocimiento del joven: CON (conocen) / NCON (no conocen).

- Integración de la familia a la propuesta: INT (se integran) / NINT (no se integran).

- Definición de estrategias de acompañamiento: FLIA (involucra familia) / PREV (de prevención) / MOT-AUT (de elevación motivación y autoestima) / SEG (de seguimiento).

- Adaptaciones curriculares: ADAP (se hacen adaptaciones) / NADAP (no se hacen adaptaciones) / DER (implica derivación externa).

Instrumentos de obtención de información y trabajo de campo

En cuanto a las técnicas de relevamiento empleadas, se seleccionaron las más adecuadas a las preguntas de investigación: entrevistas, observación y análisis de documentos generados en el centro. El total del trabajo de campo insumió 70 horas en el terreno repartidos a lo largo de ocho meses a lo largo del año escolar.

Se realizaron 30 entrevistas de tres tipos diferentes: primero se hicieron entrevistas exploratorias a tres informantes calificados, con el fin de obtener ideas para el diseño general del estudio; luego se realizaron entrevistas semiestructuradas a docentes, adscriptos $^{1}$, nueve estudiantes seleccionados y algunos integrantes de sus familias; y finalmente, se realizaron entrevistas en profundidad al equipo de dirección y a algunos docentes (los de mayor antigüedad y carga horaria).

Para seleccionar a los nueve estudiantes a los que se hizo seguimiento de los aprendizajes y se entrevistó, primero se eligieron tres grupos $\left(1^{\circ}, 2^{\circ}\right.$ y $\left.3^{\circ}\right)$ en forma aleatoria. Una vez seleccionados los tres grupos, se seleccionaron en forma intencional 9 estudiantes (tres de cada grado) de acuerdo a los siguientes criterios: tres (uno por grado) con un nivel de calificaciones muy bueno en las evaluaciones de mitad de año escolar (ninguna asignatura insuficiente); tres con calificaciones apenas aceptables (hasta tres asignaturas insuficientes); y tres con calificaciones malas (más de tres insuficientes) y predicción de repetición del curso. Resultaba particularmente relevante poder establecer alguna relación entre el tipo de rendimiento de cada estudiante y su valoración sobre las estrategias de acompañamiento de aprendizajes presentes en el centro.

A continuación, se consigna qué información fue requerida en estos dos últimos subconjuntos de entrevistas, indicado unidad de observación (quiénes fueron entrevistados) y unidad de análisis (sobre qué se indagaba).

\footnotetext{
${ }^{1}$ El adscripto juega un rol de docencia indirecta que incluye aspectos administrativos (como control de asistencia de alumnos y docentes) y de orientación, debido a su cercana relación con los alumnos. Suele permanecer todo un turno en el centro (unas 5 horas), lo cual no ocurre en el caso de muchos profesores, que permanecen menos.
} 
Entrevistas semiestructuradas:

- A docentes y adscriptos: proyecto de centro, valoración sobre el equipo de dirección, principales estrategias de enseñanza y acompañamiento, valoración sobre recursos disponibles;

- A estudiantes: descripción y valoración de estrategias de enseñanza y de acompañamiento, apoyo de las familias;

- A familias: nivel educativo de los integrantes, valoración sobre la enseñanza, formas de acompañamiento de los hijos, participación e involucramiento en la vida del centro.

Entrevistas en profundidad:

- Cuál es el rol de las familias en el centro, el perfil sociocultural de los alumnos, el trabajo en red que se hace con la comunidad donde está inserto el centro educativo, el proyecto educativo de centro, las estrategias de acompañamiento de los aprendizajes, y las formas de trabajo colaborativo.

Se realizaron observaciones del ambiente físico y de espacios comunes como recreos y espacios abiertos, así como de interacciones predominantes entre adultos y estudiantes, y entre adultos entre sí, fundamentalmente en reuniones. Se observaron clases de apoyo, donde se ponían en marcha las estrategias de acompañamiento. Estos espacios fueron relevados para obtener información sobre la dotación de recursos e instalaciones, los temas tratados en las reuniones, las pautas más salientes de relacionamiento entre todos los actores, los rasgos predominantes de la cultura del centro y del liderazgo de la dirección, y sobre todo cuáles eran las estrategias de acompañamiento de aprendizajes presentes y cómo eran puestas en marcha.

Finalmente, también se realizó análisis de documentos existentes en el centro educativo: el proyecto de centro tal cual está formulado, las actas donde se registran calificaciones de los estudiantes, y los registros que sobre cada curso tienen los profesores. A partir de los documentos se buscó información relativa al proyecto global de todo el centro educativo, a las propuestas formativas de cada profesor, al avance de los aprendizajes de los estudiantes, y a los temas tratados en las reuniones de profesores.

\section{Análisis de datos}

La información recogida en las entrevistas, las observaciones, y los documentos fue analizada siguiendo el sistema de códigos señalado líneas arriba, en torno a los cuatro conceptos centrales del trabajo: liderazgo, cultura organizacional, estrategias de acompañamiento y familias.

Se realizó triangulación para evitar sesgos, sobre todo para la validación de las entrevistas y la información de los documentos del centro: se cruzaron hallazgos con lo recogido en las observaciones. Se construyeron las respuestas a las tres preguntas de investigación agrupando hallazgos de acuerdo a los códigos y sub-códigos. Estas respuestas, que son los resultados, se incluyen a continuación. 


\section{Resultados}

El estudio identificó un conjunto de estrategias de acompañamiento de los aprendizajes que funcionan a través de diferentes roles, espacios y prácticas concretas. Se agruparon estas estrategias en cuatro subconjuntos: las de seguimiento, las orientadas a elevar la motivación y la autoestima de los alumnos, las de prevención, y las de involucramiento de las familias.

\subsection{Estrategias de seguimiento}

Estas estrategias apuntan a un mayor conocimiento individual del estudiante como tal y como persona. En este tema, el actor que juega el rol más importante es el adscripto, dada su función de interfase entre los alumnos y los docentes, su permanencia en el centro durante toda la jornada, y su cercanía con los alumnos. Es significativo que todos quienes están en contacto con los adscriptos coinciden en resaltar esta tarea: familias, profesores y alumnos. Entre las primeras, el integrante de una familia expresó: “... son muy buenas, se preocupan, te llaman, se ve que lo hacen con cariño y dedicación...”; entre los profesores subrayan "las adscriptas son una gran fortaleza, para mí es la más grande que tiene el liceo, están en todo, el día que falta una adscripta se nota, trabajan incansablemente y están muy cerca de los chiquilines...”; y entre los alumnos, el reconocimiento es similar: “... nos conocen y se preocupan por nosotros...”. Las adscriptas también son una pieza clave en los mecanismos concretos de solución de problemas. En palabras de un profesor:

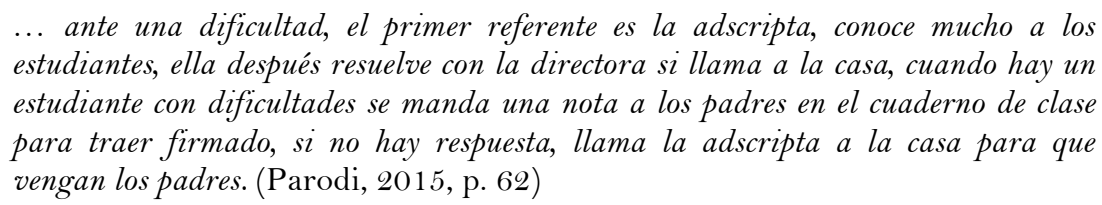

En cuanto a los espacios, además de este permanente contacto entre las adscriptas, los alumnos, y los profesores, se destaca el espacio de la Coordinación docente, como un lugar donde se puede intercambiar información relevante sobre los alumnos, donde se establece la necesidad de que asistan a clases de apoyo y donde se invita y recibe a los padres.

Entre las principales prácticas que dan forma a estas estrategias, se encontró que los profesores realizan adaptaciones curriculares para aquellos alumnos que presentan dificultades para avanzar al ritmo general. Algunas de las respuestas dadas por tres profesores ante la pregunta de si realizan adaptaciones curriculares para alumnos con necesidades educativas especiales diagnosticadas:

Sí, en las evaluaciones escritas de acuerdo a la dificultad que tenga, trabajo en computadora con los que tienen problema de escritura. Nos avisan las adscriptas en la coordinación. Cuando observo que hay alumnos con dificultades lo planteo en coordinación y comienzo a hacer evaluaciones diferenciadas. (Parodi, 2015, pp. 62-63)

Sí, en este liceo sí. La insistencia de generar pruebas diferenciadas es una exigencia de este liceo, lo pide la directora, los adscriptos nos recuerdan permanentemente, hay una hojita que dice nombre del alumno y las dificultades (para informe). Dependiendo de la dificultad, trato de identificar que entiende fácilmente y en qué áreas le cuesta más para que sea un desafio realizarlo, pero que no sea descabellado, es decir que pueda hacerlo, trato de hacer un híbrido, que sea un desafio, exigir con cuidad. (Parodi, 2015, p. 63) 
Sí, sobre todo en las evaluaciones... en el trabajo diario es más complicado porque el grupo es uno y podéis trabajar de una manera con todos y diferente con él o con ella, es más complejo. Lo que sí puedo poner es más énfasis en explicarle cosas. Igual en muchos grupos me ha pasado naturalmente algún compañero con estos chiquilines que tienen dificultades, se acerca y trata de ayudarle, lo que habla bien de un tema de solidaridad y lo mismo que hablaba hoy, no tomar esto como una competencia sino como una manera de tratar de mejorar (Asignatura Inglés). (Parodi, 2015, p. 63)

Otra práctica muy empleada se pone en marcha cuando un estudiante registra una inasistencia: de inmediato se lo requiere en el centro, y si esto no funciona, se toman medidas directas y drásticas: se lo va a buscar a su casa. Inclusive en una ocasión una adscripta fue a buscar a una alumna que no se hizo presente en una prueba, y dado que no tenía teléfono, se la fue a buscar en auto y se la trajo al centro.

También se encontró otra práctica en el terreno de los profesores, en concreto en un área crítica como es Matemática. Una docente de esta asignatura solicita los listados de alumnos que pasaron a un curso con esta asignatura pendiente de aprobación del año anterior, y los ayuda en la preparación del examen correspondiente, en forma previa al comienzo de los cursos. Se trata de una práctica excepcional en la forma de trabajo usual de los profesores de nivel secundario en el país.

\subsection{Estrategias para elevar la motivación y la autoestima}

La principal estrategia puesta en marcha dentro de este subconjunto son las clases de apoyo, también llamadas tutorías. Los docentes cumplen parte de sus horas destinadas a reuniones, desarrollando una oferta de tutorías para todas las asignaturas, de libre asistencia por parte de los alumnos, los que asisten en su gran mayoría, pues constatan su impacto positivo. Se trata de espacios de no más de 6 alumnos, con un muy buen clima de trabajo, tal cual fue observado. En las entrevistas realizadas a los alumnos, éstos le atribuyen un rol decisivo en la mejora de sus desempeños. Uno de ellos explica: “... con las tutorías... me siento más seguro, puedo preguntar lo que no entiendo, en la clase me distraigo y me pierdo." Otro alumno entrevistado expresa: “...gracias a ellas siento que he mejorado en matemática... antes no entendía nada, voy también a la de biología, repasamos lo que dimos en la clase y hacemos preguntas... somos 4, 3 de mi clase y 1 de otra clase" (Parodi, 2015, p. 67).

Desde el lugar del profesor los testimonios complementan la visión sobre las tutorías. Refiriéndose a un estudiante con importantes dificultades, un profesor expresa: “... vino todo el año a clases de apoyo y se motivó, porque es un espacio más íntimo... un día venía y hablábamos de otra cosa, logré conectar a través de la confianza” (Parodi, 2015, p. 68).

\subsection{Estrategias de prevención}

En la puesta en marcha de estas estrategias los roles protagónicos los juegan los integrantes del equipo de docencia indirecta: dirección, adscriptos y ayudantes preparadores de laboratorio. Están presentes en todos los espacios extra-aula del centro, especialmente en recreos y en las entradas y salidas del turno. Su labor preventiva y mediadora en conflictos es importante.

Una estrategia crucial para el buen funcionamiento del centro consiste en minimizar el impacto negativo de las inasistencias docentes, problema endémico de la educación del país. Cuando se pueden prever las inasistencias, se solicita a los docentes que dejen tareas listas para ser aplicadas por el personal presente. Por otra parte, el subdirector se 
dedica a solicitar suplentes en forma planificada y sistemática. Un sábado que se asistió a realizar trabajo de campo, se pudo constatar la dedicación y energía puestas por éste para solucionar las inasistencias que a la siguiente semana sabía que se producirían. El director informó que de 1.373 inasistencias que se produjeron, el 70\% se cubrió con suplentes, y $30 \%$ con tareas preparadas y supervisadas por las adscriptas presentes. Este es un tema valorado en forma positiva por las familias, dado que las inasistencias docentes son un tema de preocupación. Una las adscriptas explica cómo se organizan para cubrir algunas de las horas que quedan libres cuando se producen inasistencias docentes:

...tenemos un bibliorato con tareas que hemos recopilado, que se las ponemos, para
todos los años, pero a medida que van creciendo los estudiantes empiezan a
resistirlas y se dan cuenta que no tiene calificación y peso, se dan cuenta que no la
puso el profesor [...] si sabemos que el profesor va a faltar mañana, por ejemplo, le
pedimos que deje tarea. En algunos momentos va la directora y subdirectora a
ayudar en las horas libres. (Parodi, 2015, p. 71 )

3.4. Estrategias de involucramiento de las familias

El liceo es un espacio abierto a las familias, que tienen una presencia significativa en el centro. Los dos espacios formales en que se concreta mayor presencia de las familias y que las autoridades del centro estimulan son: las reuniones a las que se los convoca para entregarles las calificaciones de sus hijos (se realizan 7 al año), y el trabajo que algunos padres realizan de colaboración con el centro, a través de lo que se llama Asociación de Padres de Apoyo Liceal (APAL). La APAL es algo que existe en todo centro secundario del país, y funciona según el grado de involucramiento de las familias. En el caso de este centro, como se verá enseguida, se trata de una muy activa asociación de padres. Una de las adscriptas menciona que:

...trabaja muy fuerte la comisión de APAL, trabajan muy bien, se movilizan mucho,
nosotros colaboramos con todo lo que es beneficio ya sea una venta de pollo, vamos
con los chiquilines clase por clase, llevamos los tickets uno a cada uno, al otro día
pasamos a levantarlo, hacemos seguimiento alumno por alumno hasta que lo
vendan...o que lo devuelvan firmado de que en la casa efectivamente lo vieron. La
venta de pollo la tuvimos ahora, y se viene una carneada. (Parodi, 2015, p. 72)

\subsection{La cultura organizacional y el liderazgo}

En este centro se ha construido una cultura que promueve el trabajo colegiado y apunta a una visión compartida de hacia dónde se quiere ir. Se trata de un proceso que ha insumido años, y esto es posible porque el cuerpo docente es estable: la mayoría de los docentes se ha mantenido trabajando en el centro en los últimos 10 años y el director ha estado en el centro desde fines de los 1990.

El proyecto educativo de centro (elaborado en comisiones de trabajo con amplia participación) se propone el trabajo conjunto de los docentes, apuntando a un propósito fundamental que es el de la mejora continua de los aprendizajes, buscando dispositivos de retención de los alumnos. Se prioriza en él al espacio de Coordinación de los profesores, y se trabaja en proyectos interdisciplinarios. Los profesores en las entrevistas confirman que se trabaja en forma colegiada, y muchos de ellos expresan que, aunque viven lejos, eligen trabajar aquí por la forma de trabajo de alto compromiso con los aprendizajes de los alumnos.

Esta cultura ha contado para su construcción y consolidación con el liderazgo del director, una presencia, según varias fuentes, proactiva y comprometida, con un amplio 
conocimiento de toda la comunidad educativa, y con capacidad de articular personas y procesos. Estas actitudes pueden verse en su permanente recorrer clases, realizar reuniones con delegados estudiantiles, y su liderazgo en temas pedagógicos, que comenzó con el diseño del proyecto de centro y ha profundizado a lo largo del tiempo en la conducción del equipo de profesores y adscriptos.

\subsection{Presencia de las familias en el centro educativo}

El mismo proyecto de centro se plantea la importancia de involucrar a los padres en el acompañamiento de los hijos en sus aprendizajes. Para ello se propone convocar reuniones de seguimiento, talleres, entrevistas con adscriptos y profesores, y la optimización de la comunicación con las familias.

Como ya se adelantó, los dos principales espacios donde esto ocurre, son las reuniones de entrega de calificaciones y la Comisión APAL. Una de las adscriptas da cuenta de la importante participación de los padres en las reuniones (de más del 90\% todo el año), y destaca "se lo hacemos ver a los chicos, que no en todos los liceos pasa esto... nosotros realmente estamos contentos" (Parodi, 2015, p. 71). En cuanto a la APAL, las familias organizan beneficios y festejos para juntar fondos para el liceo, que se traduce en mejoras edilicias y del mantenimiento de la planta física. Obviamente, esto aumenta en forma importante el sentimiento de pertenencia de familias y alumnos con el liceo.

En las entrevistas los profesores, las adscriptas y los integrantes del equipo directivo expresan que la presencia de las familias es un rasgo central de la identidad del centro. Las familias entrevistadas confirman este ambiente positivo a su presencia, inclusivo las de los alumnos con más bajos rendimientos. Identifican como clave para este buen relacionamiento, la percepción que tienen de que en el centro existe compromiso y seguimiento de los aprendizajes de sus hijos.

\section{Discusión y conclusiones}

El estudio ha permitido ver qué sucede en un centro educativo con resultados excepcionales, en el marco de un contexto nacional que presenta problemas importantes en retención y logro de aprendizajes. Los hallazgos muestran diferentes dinámicas que tienen lugar en el centro, su tipo de liderazgo, su cultura organizacional, así como la forma que asume la presencia de las familias en él. Probablemente los hallazgos más significativos sean los relacionados con el amplio abanico de estrategias de acompañamiento de los aprendizajes identificadas y cómo el liderazgo, la cultura y las familias, se relacionan con ellas.

Los factores del nivel del centro, como el tamaño pequeño, los liderazgos con foco en los aprendizajes, la construcción de comunidades profesionales entre profesores, o el trabajo en grupo de los estudiantes que promueve la dirección, aparecen como decisivos tal como proponen otros autores (Bolívar, 2015; Faubert, 2012; Harris, 2009). También se confirma que en este centro se han neutralizado los posibles factores de ineficacia escolar señalados anteriormente como el clima negativo, las bajas expectativas, bajo compromiso y trabajo en equipo de los profesores, o de las familias, o una dirección ausente o autoritaria (Murillo, Hernández-Castilla y Martínez-Garrido, 2014). Estos factores están presentes en muchos centros que no logran buenos resultados de aprendizaje o retención de sus estudiantes. 
Destaca entre los hallazgos la permanente construcción de una cultura que se basa en la convicción de que los estudiantes pueden aprender siempre que se les provean los instrumentos adecuados, en un proactivo y comprometido liderazgo de la dirección, en altas expectativas de los profesores y sus familias, y una activa actitud de las familias que los hace involucrarse en los aprendizajes de sus hijos y en actividades organizadas en el centro, lo cual coincide con lo señalado en otros trabajos (OCDE, 2016). Se confirma la importancia de la cultura organizacional como factor decisivo en la construcción de diferentes lógicas de enfrentamiento del AEP (Tarabini et al., 2015), y dentro de ella, de las creencias y expectativas positivas de los profesores respecto a sus alumnos. De esta forma, se buscan y logran espacios específicos para realizar un seguimiento cercano de los aprendizajes. En este estudio también está presente la relevancia del rol de la dirección en el sentido de crear las condiciones para que los profesores puedan desarrollar su trabajo (Horn y Murillo, 2016) marcando una dirección clara de hacia dónde avanzar, y a la vez trabajando en soluciones concretas que garanticen la existencia de ambientes favorables a la enseñanza.

En definitiva, las estrategias desarrolladas en el centro educativo constituyen el entramado de oportunidades reales con que cuentan estos estudiantes, lo que confirma una noción de partida del estudio, que coloca el énfasis para superar el fracaso no tanto en el carácter de la escuela o del alumnado, sino en la calidad de los espacios educativos creados, como espacios de oportunidad (Rué, Amela y Buscarons, 2003).

En cuanto a las características del acompañamiento investigado, se constatan todos los aspectos señalados por Parodi (2015), excepto el registro sistemático de las intervenciones. La definición y descripción de las diferentes estrategias de acompañamiento, con las acciones específicas que implica cada una constituye uno de los principales aportes de la presente investigación: quién hace exactamente qué, cuándo y cómo. Así se puede saber cuáles son las maneras concretas y reales por las cuales un centro educativo público logra resultados excepcionales durante un período de tiempo prolongado. También el trabajo permite identificar con claridad cómo estas estrategias se basan en ciertas condiciones de la organización: un liderazgo comprometido y proactivo, una cultura de expectativas altas para los estudiantes, y una presencia activa de las familias en la vida escolar.

Estos hallazgos confirman la vigencia de varios de los conceptos clave de la literatura de la mejora de la eficacia escolar, que pone énfasis en la importancia de los liderazgos distribuidos y en las formas de trabajo colaborativo de los profesionales formadores, que ocurren en centros que se hacen cargo de los resultados de aprendizaje de sus alumnos (Bolívar, 2015; Bryk et al., 2009; Murillo y Krichesky, 2015).

Una limitación del estudio es que deja abierto el tema de la incidencia específica de cada una de las estrategias (prevención, seguimiento, mejora de la autoestima, involucramiento de las familias) en los aprendizajes, ya que aquí han sido consideradas como un conjunto. También plantea la interrogante de qué sucedería en un centro con un liderazgo tan marcado y ligado a la figura del director, si éste deja su rol y se traslada a otro centro. El sostenimiento de las estrategias construidas, así como la cultura organizacional, enfrentarían un importante desafío. Por último, la metodología del estudio de caso no permite hacer generalizaciones ya que su fin es el de profundizar en el conocimiento de un fenómeno en un contexto dado. Esto no debe ignorarse a la hora de 
valorar los aportes y las posibles transferencias de los hallazgos a otros contextos y realidades.

\section{Referencias}

Ainscow, M. (2005). Developing inclusive education systems: What are the levers for change? Journal of Educational Change, 6, 109-124. https://doi.org/10.1007/s 10833-005-1298-4

Aristimuño, A. (2015). El fracaso escolar ¿fracaso de quién? La modificación del concepto de fracaso. Revista Latinoamericana de Educación Inclusiva, 9(1), 111-126.

Aristimuño, A. (2016). Evaluaciones sobre el impacto de un programa de combate al fracaso. Revista Internacional de Medición y Evaluación de la Calidad Educativa, 3(1), 27-40.

Balsa, A. y Cid, A. (2014). Impact evaluation of a privately-managed school in a poor neighborhood in Montevideo. Páginas de Educación, 7(1), 15-31.

Bolívar, A. (2015). La mejora de la escuela. Una revisión actual. En F. J. Murillo, A. Bolívar, M. Vergara, C. Martínez-Garrido y R. Hernández-Castilla. Políticas sobre mejora de la escuela. Informes de España (Navarra y Cataluña), México, Chile e Inglaterra (pp. 8-82). Santo Domingo: MINERD.

Brookover, W. B., Beady, C., Flood, P. y Schweitzer, J. (1979). School social systems and student achievement: Schools can make a difference. Nueva York, NY: Praeger.

Bryk, A., Sebring, P. B., Allensworth, E., Luppescu, S. y Easton, J. (2009). Organizing schools for improvement: Lessons from Chicago. Chicago, IL: University of Chicago Press. https://doi.org/10.7208/chicago/9780226078014.001.0001

Cardozo, S. (2016). Trayectorias educativas en la educación media. PISA-L 2009-2014. Montevideo: Instituto Nacional de Evaluación Educativa.

Casquero, A. y Navarro, M. L. (2010). Determinantes del abandono escolar temprano en España: Un análisis por género. Revista de Educación, núm. extraordinario, 191-223.

Comisión Europea. (2010). Europa 2020. Una estrategia de crecimiento inteligente, sostenible, $e$ integrador. Bruselas: Comisión Europea.

Comisión Europea. (2013). Reducing early school leaving: Key messages and policy support. Bruselas: Comisión Europea.

De Melo, G., Failache, E. y Machado, A. (2015). Adolescentes que no asisten a ciclo básico: Caracterización de su trayectoria académica, condiciones de vida y decisión de abandono. Montevideo: Instituto de Economía.

Edmonds, R. (1979). Effective schools for the urban poor. Educational Leadership, 37(1), 15-24.

Escudero, J. M. y Martínez-Domínguez, B. (2012). Las políticas de lucha contra el fracaso escolar: ¿Programas especiales o cambios profundos del sistema y la educación? Revista de Educación, núm. extraordinario, 174-193. https://doi.org/10.4438/1988-592X.RE-2012EXT-2 11

Faubert, B. (2012). A literature review of school practices to overcome school failure. París: OECD Publishing. https://doi.org/10.1787/5k9flcwwv9tk-en

Guimaraes de Castro, H. (2011). Lead the change series. Q \& A with Helena Guimaraes de Castro. AERA Educational Change Special Interest Group, 7, 1-5.

González Berruga, M. (octubre, 2016). El papel de la orientación en la lucha contra el fracaso escolar. Comunicación presentada en el III Congreso Internacional sobre Diagnóstico y Orientación. Universidad de Jaen. 
Harris, A. (2009). Distributed leadership. Different perspectives. Londres: Springer. https://doi.org/10.1007/978-1-4020-9737-9

Harris, A. y Muijs, D. (2005). Improving schools through teacher leadership. Berkshire: Open University Press.

Horn, A. y Murillo, F. J. (2016). Incidencia de la conducción escolar sobre el compromiso de los docentes: Un estudio multinivel. Psicoperspectivas. Individuo y Sociedad, 15(2), 64-77.

Instituto Nacional de Evaluación Educativa. (2014). Informe sobre el estado de la educación en Uruguay. Montevideo: Instituto Nacional de Evaluación Educativa.

López-Yáñez, J. y Lavié, J. M. (2010). Liderazgo para sostener procesos de innovación en la escuela. Profesorado, Revista de Curriculum y Formación del Profesorado, 14, 71-92.

Martín Quintana, J. C., Alemán, J., Marchena, R. y Santana, R. (2015). Educación parental y competencias parentales para prevenir el abandono escolar temprano. Bordón, Revista de Pedagogía, 76(4), 73-92. https://doi.org/10.13042/Bordon.2015.67402

Martínez Seijo, M. L., Rayón, L. y Torrego, J. C. (2017). Las familias ante el abandono escolar. Bordón, Revista de Pedagogía, 78(2), 59-78. https://doi.org/10.13042/Bordon.2017.44617

Mena, L., Fernández Enguita, M. y Riviére, J. (2010). Desenganchados de la educación: Procesos, experiencias, motivaciones y estrategias del abandono y del fracaso escolar. Revista de Educación, núm. extraordinario, 119-134.

Murillo, F. J. (2005). La investigación sobre eficacia escolar. Barcelona: Octaedro.

Murillo, F. J. (2007). Investigación iberoamericana sobre eficacia escolar. Bogotá: Andrés Bello.

Murillo, F. J. y Krichesky, G. (2015). Mejora de la escuela: medio siglo de lecciones aprendidas. REICE. Revista Iberoamericana sobre Calidad, Eficacia y Cambio en Educación, 13(1), 69-102.

Murillo, F. J. Hernández-Castilla, R. y Martínez-Garrido, C. (2014). Factores de ineficacia escolar. REICE. Revista Iberoamericana sobre Calidad, Eficacia y Cambio en Educación, 12(1), 103-118.

Murillo, F. J., Bolívar, A. Vergara, M., Martínez-Garrido, C. y Hernández-Castilla, R. (2015). Políticas sobre mejora de la escuela. Informes de España (Navarra y Cataluña), México, Chile e Inglaterra. Santo Domingo: MINERD.

OCDE. (2016). Estudiantes de bajo rendimiento: Por qué se quedan atrás y cómo ayudarles a tener éxito. París: OCDE.

Parodi, J. P. (2015). Las características del acompañamiento escolar en un centro educativo con altos niveles de promoción (Tesis de Máster). Universidad Católica del Uruguay, Montevideo.

Pedró, F. (2012). Políticas públicas sobre apoyo y refuerzo educativo. Evidencias internacionales. Revista de Educación, núm. extraordinario, 22-45.

Pont, B. (2014). Lead the change series. Q \& A with Beatriz Pont. AERA Educational Change Special Interest Group, 35, 1-6.

Rivas, A. (2015). América Latina después de PISA. Lecciones aprendidas de la educación en siete países (2000-2015). Buenos Aires: CIPPEC.

Rué, J., Amela, M. y Buscarons, M. (2003). El absentismo escolar como reto para la calidad. Madrid: CIDE.

Rumberger, R. M. (2011). Dropping out. Why students drop out of high school and what can be done about it. Boston, MA: Harvard University Press. https://doi.org/10.4159/harvard.9780674063167 
Santos-Rego, M. A., Godás-Otero, A. y Lorenzo-Moledo, M. (2012). El estatus del alumnado repetidor y no repetidor en una muestra de estudiantes españoles y latinoamericanos. Un estudio sobre los determinantes de sus logros académicos. Estudios sobre Educación, 23, 4362.

SITEAL. (2009). ¿Por qué los adolescentes dejan la escuela? Motivos de la deserción en la transición del primario al secundario. Recuperado de http://www.siteal.iipe-oei.org

Tarabini, A., Curran, M., Montes, A. y Parcerisa, L. (2015). El rol de los centros educativos en la prevención del abandono escolar. Una aproximación desde la perspectiva micropolítica. Educacao, Sociedade \& Culturas, 45, 121-141.

Yin, R. (2013). Case study research. Design and methods. Thousand Oaks, CA: Sage.

\section{Breve CV de los autores}

\section{Adriana Aristimuño}

Licenciada y doctora en ciencias de la educación (Universidad Católica de L $f$ lovaina, Bélgica). Ha realizado el seguimiento de políticas de educación media en la educación pública de Uruguay. Profesora Titular en carreras de grado y postgrado, formadora de investigadores y gestora en la Universidad Católica del Uruguay, donde ha sido Decana de la Facultad de Ciencias Humanas e integrante de su Consejo Directivo. Profesora invitada en universidades de Argentina, Chile, Colombia, México y Perú. Sus líneas de investigación son la política educativa, los procesos de cambio y las estrategias de mejora, con foco en la educación media. Ha realizado trabajos como consultora en temas de educación para BID, el IIPE de UNESCO y UNICEF y tiene más de treinta publicaciones en sus temas de interés. Integra el Sistema Nacional de Investigadores del Uruguay. ORCID ID: 0000-0003-2597-7603. Email: aaristim@ucu.edu.uy

\section{Juan Pablo Parodi}

Profesor titulado de la asignatura Biología de enseñanza secundaria, Postgraduado y Magister en Orientación educativa por la Universidad Católica del Uruguay. Ha sido docente de aula en Biología en centros de educación secundaria públicos y privados, y en programas de rescate de desertores de la enseñanza secundaria pública. Ha desempeñado roles de orientación y asesoramiento de adolescentes, y actualmente es director de un centro educativo de educación secundaria (niveles básico y superior). ORCID ID: O0OOoo03-2497-8137. Email: parodijuanpablo@gmail.com 